\title{
Editorial: Our vision for PRX Quantum - a new home for a vibrant community
}

It is our pleasure to introduce PRX Quantum to the quantum information science and technology research community. PRX Quantum is born to meet this community's desire for a new home within the Physical Review family of journals: one that is highly selective and showcases the best research in the field. The 'PRX' in its name indicates its connection to Physical Review X (PRX) and the excellence that journal has come to represent. PRX Quantum is designed to represent the vibrant and dynamic research of this field, enhance the visibility of the best results through open access, promote the broad exchange of ideas, and inspire new generations.

The rise of quantum information science over the past several decades has been truly remarkable. In its earliest days, a small community of researchers was exploring how the exotic predictions of quantum physics, such as superposition and entanglement, might be put to use. The Physical Review family of journals has covered this research since those very beginnings. Several seminal papers that have profoundly impacted the way we view 'information' in a quantum world are found in the pages of Physical Review Letters (PRL) and Physical Review A.

As the field matured, it has greatly expanded and embraced new communities. Concepts and ideas from quantum information now also regularly appear in PRX and several of the other Physical Review journals. As the field continues its rapid development, we expect that novel directions may soon appear in other specialized journals as well. And while much of the early research was theoretical, the field now encompasses vibrant experimental as well as theoretical efforts, and has reached a level of maturity in which the potential technological impact on society is no longer science fiction. The research community, once an esoteric corner in a few university departments, is now a thriving academic discipline accompanied by major research communities in industrial labs and tech hubs. We are living in the so-called second quantum revolution.

There is a clear need to provide a home for peer reviewed research that bridges the different disciplines working within quantum information science and technology. We aim to welcome the full spectrum of researchers working on this topic, from computer scientists to materials scientists, theoretical physicists to physical chemists, mathematicians, engineers, and beyond. To best represent this diverse community and keep in close contact with them, we judiciously chose the composition of our Editorial Board. We are excited and proud to count on an outstanding, diverse and global team of Editorial Board members, from academy and industry, that will help us to shape this journal.

Building on the excellence of PRX, PRX Quantum is a highly-selective journal featuring research of outstanding and lasting impact. This selectivity comes in response to community demand: the flagship journals PRX and PRL experience strong interest from quantum information science and technology fields, and researchers are clearly seeking a venue to publish topical research articles with similar high standards and the trusted Physical Review experience. Our expert Editorial Board members, as well as our many valued referees from the research communities, will robustly maintain these high standards in partnership with our authors. As editors, our guiding principle is that all parties in the review process can build an open and honest dialogue, working together to select the very best research results in quantum. To increase the visibility of the published results, the journal has adopted a fully open access model.

In addition to publishing research articles, we are happy to serve the community with two other article types: Tutorials and Perspectives. Tutorials stem from a recognition that we should urgently educate the next generation of quantum scientists. They aim to provide a hands-on guide for newcomers, and will especially benefit $\mathrm{Ph} . \mathrm{D}$. students and postdocs entering relevant fields, as well as anyone interested in learning more about a subject in quantum beyond their own area of expertise. On a different end of the spectrum, Perspectives are forward-looking articles by research visionaries. They are a venue for inspiring our readers and for sharing the excitement. They should also aim to bridge the diverse communities

Published by the American Physical Society under the terms of the Creative Commons Attribution 4.0 International license. Further distribution of this work must maintain attribution to the author(s) and the published article's title, journal citation, and DOI. 
working in the broader field of quantum information science and technology. We hope they will convey the challenges and the potential for scientific, technological, and engineering advances that will help to overcome key obstacles.

We are looking forward to seeing your next exciting results published in PRX Quantum and invite you to build this journal together with us.

Stephen Bartlett
Lead Editor

Katiuscia N. Cassemiro co-Managing Editor

Stojan Rebic

co-Managing Editor

(Q) Published 3 September 2020

DOI: 10.1103/PRXQuantum.1.010002 